\title{
CARTAN SUBALGEBRAS OF JORDAN ALGEBRAS
}

\author{
N. JACOBSON ${ }^{11}$
}

\section{Dedicated to the memory of TADasi Nakayama}

In this paper we shall give a definition of an analogue for Jordan algebras of the classical notion of a Cartan subalgebra of a Lie algebra. This is based on a notion of associator nilpotency of a Jordan algebra. A Jordan algebra $\mathfrak{Y}$ is called associator nilpotent if there exists a positive (odd) integer $M$ such that every associator of order $M$ formed of elements of $\xi$ is $0(\S 2)$. If $a, b \in$ $\Im$ then we set $R_{a, b}=R_{a} R_{b}-R_{a \cdot b}$ where $R_{a}$ is the mapping $x \rightarrow x \cdot a$ (product of $x$ and $a$ ) in $\mathfrak{Y}$. An element $a$ is called associator nilpotent in $\mathfrak{I}$ if all the operators of the form $R_{a^{\cdot i}, a^{\cdot j}}$ are nilpotent. We prove an analogue of Engel's theorem on Lie algebras to the effect that a finite dimensional Jordan algebra $\mathfrak{F}$ (with 1) over an infinite field is associator nilpotent if and only if every element of $\mathfrak{\Im}$ is associator nilpotent (Theorem 4). If $\mathfrak{\Re}$ is an associator nilpotent subalgebra of the finite dimensional Jordan algebra $\mathfrak{Z}$ then the Lie algebra $\mathfrak{L}_{\mathfrak{S}}(\Re)$ of linear transformations in $\mathfrak{Y}$ generated by the linear transformations in $\mathfrak{Y}$ of the form $R_{b, c}, b, c \in \Re$ is nilpotent. We define a Cartan subalgebra of $\Im$ to be an associator nilpotent subalgebra $\Re$ of $\mathfrak{\Im}$ such that the Fitting null component

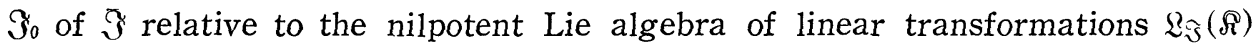
coincides with $\Re(\S 5)$.

If $a \in \Im$ and $\operatorname{dim} \Im=n$ then we set $3 a=\left\{z \in \Im \mid z\left(R_{a^{\cdot i, a} \cdot a^{\prime}}\right)^{n}=0, i, j=0,1,2, \ldots\right\}$. The element $a \in \mathfrak{Y}$ is called associator regular in $\Im$ if $\operatorname{dim} 3 a$ is minimal. If the base field is infinite then $3 a$ is a Cartan subalgebra for any associator regular element $a$ (Theorem 6 ). If the base field is algebraically closed of characteristic 0 then any two Cartan subalgebras are conjugate in a strong sense (Theorem 7).

As applications of our results we obtain a formula for the generic trace

Receired July 7, 1965.

1) This research has been supported by the Air Force Office of Scientific Research under Grant AF-AFOSR-402-64. 
and we give a proof of the trace criterion for separability of a finite dimensional Jordan algebra, which is independent of the determination of the finite dimensional simple Jordan algebras over an algebraically closed field (§7). In $\S 1$ we collect the results on Jordan algebras which are needed in the remainder of the paper.

1. Preliminaries. In this paper all algebras are algebras over fields of characteristic not two, all associative algebras are assumed to have identity elements, and the usual conventions for such algebras will be adopted: subalgebras will be assumed to contain 1, homomorphisms to map 1 into 1 , etc. Beginning with $\S 2$ we shall adopt these conventions also for Jordan algebras. However, we do not do this in the present section. We denote the product in a Jordan algebra by $a . b$, so, the defining identities are $a \cdot b=b . a,\left(a^{\cdot 2} \cdot b\right) \cdot a=a^{\cdot 2} \cdot(b \cdot a)$ where $a^{-2}=a . a$. These have the following consequences:

$$
\begin{aligned}
& (x . a) \cdot(b \cdot c)+(x \cdot b) \cdot(a \cdot c)+(x \cdot c) \cdot(a \cdot b) \\
& \quad=(x \cdot(b \cdot c)) \cdot a+(x \cdot(a \cdot c)) \cdot b+(x \cdot(a \cdot b)) \cdot c \\
& ((x \cdot a) \cdot b) \cdot c+((x \cdot c) \cdot b) \cdot a+x \cdot((a \cdot c) \cdot b) \\
& \quad=(x \cdot a) \cdot(b \cdot c)+(x \cdot b) \cdot(a \cdot c)+(x \cdot c) \cdot(a \cdot b) \cdot{ }^{2)}
\end{aligned}
$$

If we denote the linear mapping $x \rightarrow x . a$ by $R_{a}$ then $\left[R_{a}, R_{a .2}\right]=0$ where $[A, B]=A B-B A$, and (1) and (2) are equivalent to

$$
\begin{gathered}
{\left[R_{a}, R_{b . c}\right]+\left[R_{b}, R_{a . c}\right]+\left[R_{c}, R_{a . b}\right]=0} \\
R_{a} R_{b} R_{c}+R_{c} R_{b} R_{a}+R_{(a . c) . b}=R_{a} R_{b . c}+R_{b} R_{a . c}+R_{c} R_{a . b} .
\end{gathered}
$$

Jordan algebras are power associative, that is, if we define $a^{\cdot k}$ by $a^{\cdot 1}=a, a^{\cdot k}=$ $a^{\cdot k-1} \cdot a, k=2,3, \ldots$, then $a^{\cdot k} \cdot a^{\cdot l}=a^{\cdot k+l}$. We recall also that if $\mathfrak{G}$ is a Jordan algebra over a field $\Phi$ and $I I$ is an extension field of $\Phi$ then the extension algebra $\Im_{n}=\Pi \otimes_{\Phi} \Im$ is Jordan.

Let 3 be a Jordan algebra with an identity element 1 and suppose $1=$ $\sum_{1}^{n} e_{i}$ where the $e_{i}$ are orthogonal idempotent elements $\left(e_{i}^{2}=e_{i}, e_{i} . e_{j}=0\right.$ if $\left.i \neq j\right)$. Let $\mathfrak{Y}_{i i}=\left\{x_{i i} \mid x_{i i} . e_{i}=x_{i i}\right\}$ and $\mathfrak{\Im}_{i j}=\left\{x_{i j} \mid x_{i j} . e_{i}=\frac{1}{2} x_{i j}=x_{i j} . e_{j}\right\}$ if $i \neq j=1, \ldots, n$. Then

2) The results stated in this section without proof or reference can be found in Albert [2]. 


$$
\mathfrak{F}=\sum_{i=j} \oplus \Im_{i j}
$$

We call the $\Im_{i j}=\Im_{j i}$ the Peirce spaces of $\Im$ relative to the orthogonal idempotents $e_{i}$ and (3) the Peirce decomposition of $\Im$ relative to these idempotents. We have the following multiplication table for the Peirce spaces:

$$
\begin{aligned}
& \Im_{i l}^{\cdot 2} \subseteq \Im_{i i}, \quad \Im_{i i} \cdot \Im_{j j}=0 \text { if } i \neq j \\
& \Im_{i j} \cdot \Im_{i i} \subseteq \Im_{i j}, \quad \Im_{i j}^{2} \subseteq \Im_{i i}+\Im_{j j} \text { if } i \neq j \\
& \Im_{i j} \cdot \Im_{j k} \subseteq \Im_{i k}, \quad \Im_{i i} \cdot \Im_{j k}=0, \Im_{i j} \cdot \Im_{k l}=0 \text { if } i, j, k, l \text { are unequal. }
\end{aligned}
$$

We shall call a Jordan (or associative) algebra $\Im$ over a field $\emptyset$ almost nil if $\mathfrak{Y}$ has an identity 1 and $\}=\emptyset 1+\mathfrak{R}$ where $\mathfrak{R}$ is a nil ideal. If $\mathcal{Y}$ is a Jordan algebra with 1 such that every element of $\Im$ has the form $\alpha 1+z$ where $\alpha \in \Phi$ and $z$ is nilpotent, then $\mathcal{G}$ is almost nil (Albert [3] p. 514, Jacobson [4], McCrimmon [1]). This implies that if $\Im$ is finite dimensional with 1 over an algebraically closed field and $1=\sum_{1}^{r} e_{i}$ where the $e_{i}$ are orthogonal idempotents $\neq 0$ and are primitive in the sense that we cannot write $e_{i}=e_{i}^{\prime}+e_{i}^{\prime \prime}$ where $e_{i}^{\prime} \neq 0, e_{i}^{\prime \prime} \neq 0$ and $e_{i}^{\prime} \cdot e_{i}^{\prime \prime}=0$, then the Peirce spaces $\Im_{i i}$ are almost nil Jordan algebras: $\mathfrak{\Im}_{i i}=\emptyset e_{i}+\mathfrak{N}_{i}, \mathfrak{\Re}_{i}$ a nil ideal in $\mathfrak{\Im}_{i i}$. If $\mathfrak{\Im}$ is finite dimensional then the following three conditions on $\Im$ are equivalent: (1) $\Im$ is solvable (2) $\mathfrak{I}$ is a nil algebra (3) $\Im$ is nilpotent, in the sense that there exists an integer $N$ such that every product of $N$ elements (in any association) of $\Im$ is 0 . A finite dimensional Jordan algebra contains a maximal solvable ideal $s$ called the radical of $\mathcal{S}$. If $\mathcal{G}$ is finite dimensional and $\Re$ is a non-zero solvable ideal then $\Re$ contains an ideal $\mathscr{R}^{\prime} \neq \Re$ of $\mathfrak{\Im}$ such that $\Re^{2} \subseteq \mathscr{R}^{\prime}$ (Penico [1], p. 408).

A finite dimensional Jordan algebra is called semi simple if the radical $\Im=0$. The algebra $\Im / \Im$ is semi-simple. If $\Im$ is semi-simple then $\Im$ has an identity element and is a direct sum of simple ideals (Albert [3]). If $\mathfrak{F}$ is a finite dimensional simple Jordan algebra over an algebraically closed field $\mathscr{D}$ then either $\Im=\emptyset 1$ or $\Im$ contains $r>1$ primitive orthogonal idempotent elements $e_{i} \neq 0$. Moreover, the Peirce spaces $\Im_{i j}, i \neq j$, determined by these elements are non-zero (Albert [3], Jacobson [4]).

Let $\mathfrak{\Im}$ be an arbitrary Jordan algebra and let $\mathfrak{I}(\mathfrak{I})=\emptyset \oplus \mathfrak{I} \oplus(\mathfrak{I} \otimes \mathfrak{S}) \oplus(\mathfrak{S}$ $\otimes \mathfrak{\Im} \otimes \oplus \cdots$ be the tensor algebra based on the vector space $\mathfrak{I}, \mathfrak{R}$ the ideal in $\mathfrak{I}(\mathfrak{S})$ generated by all elements of the form 


$$
\begin{gathered}
a \otimes a^{2}-a^{2} \otimes a \\
a^{\cdot 2} . b+2 a \otimes b \otimes a-b \otimes a^{2}-2 a \otimes a . b,
\end{gathered}
$$

where $a, b \in \mathfrak{I}$. The associative algebra $\mathfrak{U}(\mathfrak{I})=\mathfrak{I}(\mathfrak{I}) / \mathfrak{R}$ will be called the universal multiplication envelope of the Jordan algebra $\mathfrak{J}^{3}{ }^{3}$ If $a \in \mathfrak{Y}$ we set $a^{r}=a+\Re$ in $\mathfrak{H}(\Im) . \mathfrak{H}(\Im)$ and the mapping $a \rightarrow a^{r}$ have the following universal mapping property: Let $a \rightarrow a^{\rho}$ be a linear mapping of $\mathcal{Y}$ into an associative algebra $\mathfrak{A}$ such that $\left[a^{\rho}, a^{.{ }^{\rho}}\right]=0$ and $\left(a^{.2} . b\right)^{\rho}+2 a^{\rho} b^{\circ} a^{\rho}=b^{\rho} a^{.2 \rho}+2 a^{\rho}(a . b)^{\rho}$ then there exists a unique homomorphism of $\mathfrak{u}(\mathfrak{S})$ into $\mathfrak{U}$ such that $a^{r} \rightarrow a^{\rho}$. It follows from this that if $\Re$ is an ideal in $\mathfrak{J}$ and $\mathfrak{B}$ is the ideal in $\mathfrak{U}(\mathfrak{G})$ generated by $\Re$ then we have an isomorphism of $\mathfrak{U}(\Im / \Re)$ onto $\mathfrak{H}(\mathfrak{\Im}) / \mathfrak{B}$ mapping $(a+\Re)^{r}$, $a \in \mathfrak{F}$, into $a^{r}+\mathfrak{B}$ (cf. Jacobson, Lie Algebras, Th. 5.1 (4), p. 153). It is known that $\mathfrak{H}(\Im)$ is finite dimensional if $\Im$ is finite dimensional (Jacobson [2], p. 519).

Let $\mathcal{Y}$ be a subalgebra of the Jordan algebra $\Im^{\prime}$ and let $R_{a}, a \in \mathcal{Y}$, be the multiplication $x \rightarrow x . a$ in $\Im^{\prime}$. Then $a \rightarrow R_{a}$ is a linear mapping of $\Im$ into $\operatorname{Hom}_{\Phi}\left(\Im^{\prime}\right.$, $\left.3^{\prime}\right)$ and we have $\left[R_{a}, R_{a \cdot 2}\right]=0$ and $2 R_{a} R_{b} R_{a}+R_{a \cdot 2 . b}=2 R_{a} R_{a . b}+R_{b} R_{a \cdot 2}$. Hence we have a homomorphism of $\mathfrak{u}(\mathfrak{\Im})$ onto the subalgebra $\mathbb{E}$ of $\operatorname{Hom}_{\mathbb{P}}\left(\mathfrak{I}^{\prime}, \mathfrak{\Im}^{\prime}\right)$ generated by the $R_{a}, a \in \Im$. Let $\Im$ be finite dimensional and let $\Re$ be a solvable ideal in $\Im$. Then we wish to show that the elements $R_{b}, b \in \mathfrak{R}$ (acting in $\mathfrak{S}^{\prime}$ ), generate a nilpotent ideal in (5.) In view of the homomorphism we have just noted this will follow from the following

Theorem 1. Let $\mathfrak{\Im}$ be a finite dimensional Jordan algebra, $\mathbb{R}$ a solvable ideal in $\mathfrak{\Im}$. Then the image $\AA^{r}$ of $\mathfrak{R}$ in $\mathfrak{M}(\Im)$ generates a nilpotent ideal in $\mathfrak{H}(\Im)$.

We shall prove this result by induction on the dimensionality $\operatorname{dim} \Omega$ and we may assume $\mathscr{R} \neq 0$. Let $\mathscr{R}^{\prime}$ be an ideal of $\Im$ properly contained in $\mathscr{R}$ such that $\mathscr{R}^{2} \subseteq \mathbb{R}^{\prime}$. Then $\mathfrak{R}^{\prime r}$ generates a nilpotent ideal $\mathfrak{B}$ in $\mathfrak{U}(\mathfrak{S})$ and we have the isomorphism noted above of $\mathfrak{H}\left(\mathfrak{\Im} / \mathfrak{N}^{\prime}\right)$ onto $\mathfrak{H}(\mathfrak{\Im}) / \mathfrak{B}$. In view of this it suffices to show that the image of $\mathscr{R} / \mathscr{N}^{\prime}$ in $\mathfrak{U}\left(\Im / \Re^{\prime}\right)$ generates a nilpotent ideal. Accordingly, it is enough to prove the theorem for the case $\AA^{2}=0$. In this case we base the proof on the following two lemmas.

3) This is a slightly different definition from that of the universal associative algebra of representations of $\Im$ given in Jacobson [2] and [3]. The modification has been made to take care of the characteristic three case. The results of Jacobson [2] and [3] carry over without change to the present situation.

4) For characteristic 0 this is proved in Jacobson [2], p. 522. 
Lemma 1. Let $\Im$ be a finite dimensional Jordan algebra such that $\mathfrak{\Im}^{2}=0$. Then the image $\mathfrak{Y}^{r}$ of $\mathfrak{Y}$ in $\mathfrak{u}(\mathfrak{\xi})$ generates a nilpotent ideal.

Proof. Since any subspace of $\mathfrak{\Im}$ is an ideal the inductive argument we have just used gives a reduction to the case in which $\operatorname{dim} \Im=1$. Then $\mathfrak{F}=\emptyset e$. The general relation

$$
\left(a^{\cdot 2} \cdot b\right)^{r}+2 a^{r} b^{r} a^{r}=b^{r}\left(a^{.2}\right)^{r}+2 a^{r}(a \cdot b)^{r}
$$

in universal multiplication envelopes gives the relation $\left(e^{r}\right)^{3}=0$ in $\mathfrak{H}(\mathcal{Y})$. Since

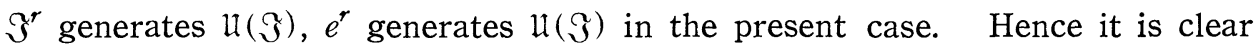
that $\Im^{r}=(\varpi e)^{r}$ generates a nilpotent ideal in $\mathfrak{u}(\Im)$.

Lemma 2. Let $\Im$ be a finite dimensional Jordan algebra, $\Re$ a non-zero ideal in $\Im$ such that $\AA^{2}=0$. Let $\left(e_{1}, e_{2}, \ldots, e_{n}\right)$ be a basis for $\Im$ such that $\left(e_{1}, e_{2}\right.$, $\left.\ldots, e_{m}\right)$ is a basis for $\Re, k$ a positive integer. Then any monomial in $\mathfrak{U}(\xi)$ of the form $e_{j_{1}}^{r} e_{j_{2}}^{r} \cdots e_{j_{l}}^{r}$ in which $k+m$ of the $j$ 's are in the range $I=\{1,2, \ldots, m\}$ is a linear combination of monomials of the form $e_{i_{1}}^{r} e_{i_{2}}^{r} \cdots e_{i_{k}}^{r} \cdots$ where $i_{1}, \ldots$, $i_{k} \in I$.

Proof. This is clear if $l=k+m$; hence we may use induction on the formal degree $l$ of the monomial. Now suppose the first $h$ subscripts $j$ in the given monomial $M=e_{j_{1}}^{r} e_{j_{2}}^{r} \cdots e_{j l}^{r}$ are in $I$ but the $h+1$-st subscript is not. Then the assertion holds if $h \geq k$, so for a given $l$ we may use a downward induction on the integer $h$ and we may assume $h<k$. Then $M$ has the form

$$
\cdots e^{r} \cdots e^{r} \cdots
$$

where $e$ is one of the $e_{i}, i \in I$, and the first displayed $e^{r}$ in (7) occurs after the $h+1$-st place. Let $B$ denote the space of linear combinations of monomials of the form $e_{i_{1}}^{r} e_{i_{2}}^{r} \cdots e_{i_{k}}^{r} \cdots$ where $i_{1}, i_{2}, \ldots, i_{k} \in I$. Suppose first that there is just one $e_{j}^{r}$ between the indicated $e^{r}$ in (7). Then (6) for $a=e, b=e_{j}$ gives the relation $e^{r} e_{j}^{r} e^{r}=e^{r}\left(e . e_{j}\right)^{r}$. Since $e . e_{j} \in \Re$ and $e . e_{j}=0$ if $e_{j} \in \Re$ substitution of the relation just given in $(7)$ and the induction hypothesis on $l$ implies that $M \in \mathfrak{B}$. Next assume that the indicated $e^{r}$ in (7) are consecutive. We have the following relation in universal multiplication envelopes which is a consequence of $(6)$ :

$$
\begin{aligned}
a^{r} b^{r} c^{r}= & -c^{r} b^{r} a^{r}-((a . c) \cdot b)^{r}+a^{r}(b . c)^{r} \\
& +b^{r}(a . c)^{r}+c^{r}(a . b)^{r} .
\end{aligned}
$$


Setting $b=c=e$ and $a=e_{j}$ where $e_{j}^{r}$ precedes the first $e^{r}$ in (7) we obtain $e_{j}^{r}\left(e^{r}\right)^{2}=-\left(e^{r}\right)^{2} e_{j}^{r}+2 e^{r}\left(e_{j} . e\right)^{r}$. A succession of replacements of this type shows that $M \equiv \pm M^{\prime}(\bmod B)$ where $M^{\prime}$ has formal degree $l$, has $k+m$ factors of the form $e_{i}^{r}, i \in I$, and has $h+2$ such factors at the beginning of $M^{\prime}$. The induction on $h$ can be invoked to conclude that $M^{\prime} \in \mathfrak{B}$. Hence $M \in \mathfrak{B}$. Finally, suppose we have at least two $e_{j}^{r}$ between the displayed $e^{r}$ in (7). Then we can use (8) with $c=e$ to show that $M \equiv-M^{\prime}(\bmod B)$ where $M^{\prime}$ is obtained from $M$ by moving the second $e^{r}$ two places to the left. A succession of moves of this type shows that $M \equiv \pm N(\bmod \mathfrak{B})$ where $N$ is obtained from $M$ by moving the second $e^{r}$ either next to the first one or to the position two places after the first one. Then $N \in \mathfrak{B}$ by the first two cases. Hence $M \in \mathfrak{B}$ in all cases.

We can now complete the proof of Theorem 1 for the case $\AA^{2}=0$. By Lemma 1 and the universal mapping property of $\mathfrak{u}(\Re)$ there exists a positive integer $N$ such that the product of any $N$ elements of the form $e^{r}, e \in \Re$ is 0 . By Lemma 2 it follows that the product of any elements $a^{r}, a \in \mathfrak{F}$, which includes $N+m(m=\operatorname{dim} \Re)$ elements of $\Re$ is 0 . This implies that if $\mathfrak{B}$ is the ideal in $\mathfrak{l}(\mathfrak{\Im})$ generated by $\mathfrak{R}^{r}$ then $\mathfrak{B}^{N+m}=0$.

2. Associator nilpotent Jordan algebras. From now on we assume that all the Jordan algebras under consideration are finite dimensional and contain 1. The usual conventions for algebras with 1 are adopted. In particular, subalgebras necessarily contain 1 .

Let $\Im$ be a Jordan algebra over the field $\emptyset$ (finite dimensional with 1 ). If $a, b, c \in \mathfrak{Y}$ we write $[a, b, c]$ for the associator $(a . b) . c-a .(b . c)$. This defines a trilinear composition in $\mathfrak{Z}$ which can be iterated to define $n$-linear compositions for any positive odd integer $n$. Let $n$ be a positive odd integer, $\left(a_{1}, a_{2}\right.$, $\left.\ldots, a_{n}\right)$ an ordered set of elements $a_{i} \in \mathcal{Y}$. Then we define an associator of order $n A_{n}\left(a_{1}, \ldots, a_{n}\right)$ inductively by $A_{1}\left(a_{1}\right)=a_{1}$,

$$
\begin{array}{r}
A_{n}\left(a_{1}, \ldots, a_{n}\right)=\left[A_{n_{1}}\left(a_{1}, \ldots, a_{n_{1}}\right), A_{n_{2}}\left(a_{n_{1}+1}, \ldots, a_{n_{1}+n_{2}}\right),\right. \\
\left.A_{n_{3}}\left(a_{n_{1}+n_{2}+1}, \ldots, a_{n_{1}+n_{2}+n_{3}}\right)\right]
\end{array}
$$

where $n=n_{1}+n_{3}+n_{3}, n_{i}$ a positive odd integer and $A_{n_{i}}(\cdots)$ is an associator of order $n_{i}$. We shall call $\xi$ associator nilpotent if there exists a positive odd integer $M$ such that every associator of order $M$ formed of elements of $\mathfrak{I}$ is 0 . 
The minimum $M$ with this property is called the index of associator nilpotency of 3 . It is clear that subalgebras, homomorphic images and direct sums of associator nilpotent Jordan algebras are associator nilpotent. Also since the higher associator compositions are multilinear it is clear that $\Im$ is associator nilpotent if and only if $\Im_{\Pi}=\Pi \otimes_{\Phi} \mathfrak{\Im}$ is associator nilpotent for any extension field $\Pi / \emptyset$. This remark will permit us to reduce considerations on associator nilpotency to the case of an algebraically closed field. For these we have the following important criterion.

TheOREм 2. A Jordan algebra over an algebraically closed field is associator nilpotent if and only if it is a direct sum of ideals which are almost nil algebras.

Proof. To prove the sufficiency it is enough to show than any almost nil

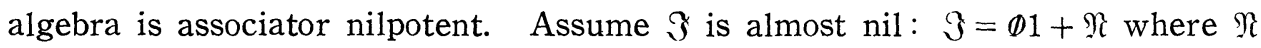
is a nil subalgebra. Since the higher associator compositions are multilinear and since any higher associator involving 1 is 0 , to prove associator nilpotency it is enough to show that there is an odd integer $M$ such that every associator of order $M$ of elements $z_{i} \in \mathfrak{N}$ is 0 . This is clear since $\mathfrak{N}$ nil implies that $\mathfrak{R}$ is nilpotent. Conversely, assume $\mathfrak{Y}$ is associator nilpotent and let $\Im=\sum_{i=j} \Im_{i}$ be a Peirce decomposition of $\mathfrak{F}$ relative to a set of orthogonal primitive idempotent elements $e_{i} \neq 0, i=1,2, \ldots, r$, such that $\sum e_{i}=1$. Then every $\Im_{i i}$ is an almost nil algebra with identity element $e_{i}$. Let $a_{i j} \in \Im_{i j}, i \neq j$. Then $\left[a_{i j}, e_{i}, e_{j}\right]=\left(a_{i j} . e_{i}\right) . e_{j}-a_{i j} .\left(e_{i}, e_{j}\right)=\frac{1}{4} a_{i j}$. Iteration of this gives

$$
\left[\cdots[a_{i j}, \overbrace{\left.\left.\left.e_{i}, e_{j}\right], e_{i}, e_{j}\right], \ldots, e_{i}, e_{j}\right]}^{k}=\left(\frac{1}{4}\right)^{k} a_{i j} .\right.
$$

Since $\Im$ is associator nilpotent, $k$ can be chosen so that the left-hand side is 0 . Hence $a_{i j}=0$ and so $\Im_{i j}=0$ if $i \neq j$. Since $\Im_{i t} . \Im_{j j}=0$ for $i=j$ it is now clear that the $\Im_{i i}$ are ideals and $\Im=\Im_{11} \oplus \Im_{22} \oplus \cdots \oplus \Im_{r r}$ is a direct sum of almost nil ideals.

We prove next the following necessary (but not sufficient) condition for associator nilpotency.

THEOREM 3. If $\mathfrak{\Im}$ is associator nilpotent then $\mathfrak{\Im} / \mathbb{S}$ is associative for $\mathbb{S}$ the radical of $\Im$.

Proof. Since $\Im / \widetilde{S}$ is associator nilpotent it is enough to show that if $\Im$ 
is associator nilpotent and semi-simple then $\mathfrak{Y}$ is associative. Since a semisimple Jordan algebra is a direct sum of simple ideals we may assume $\Im$ simple. Then the center $\Gamma$ of $\Im$ is a field and $\Im$ over $\Gamma$ is central simple. Also it is clear that $\mathfrak{F}$ over $\Gamma$ is associator nilpotent. Hence we may assume $\mathfrak{Y}$ is central simple. Then $\Im_{\Omega}$ is simple for $\Omega$ the algebraic closure of the base field. We have noted in $\S 1$ that if $\operatorname{dim} \Im_{\Omega}>1$, then $\Im_{\Omega}$ contains $r>1$ primitive orthogonal idempotent $e_{i} \neq 0$ such that $\sum e_{i}=1$. Also the Peirce spaces $\Im_{i j}$, $i \neq j$, determined by the $e_{i}$ are $\neq 0$. Then $\left.\left[\cdots\left[a_{i j}, e_{i}, e_{j}\right], e_{i}, e_{j}\right] \cdots e_{i}, e_{j}\right]=$ $\left(\frac{1}{4}\right)^{k} a_{i j} \neq 0$ for $a_{i j} \neq 0$ in $\Im_{i j}$. This contradicts the associator nilpotency of $\Im$. Hence $\operatorname{dim} \mathfrak{\Im}_{\Omega}=1$ so $\Im_{\Omega}$ is associative. Hence $\Im_{\text {is }}$ issociative.

The following example shows that the converse of Theorem 2 is false.

Example. Let $\Im$ be the Jordan algebra of triangular matrices $\left(\begin{array}{ll}\alpha & \gamma \\ 0 & \beta\end{array}\right)$ where $\alpha, \beta, \gamma \in \emptyset$ and the multiplication is $a \cdot b=\frac{1}{2}(a b+b a)$. The radical $\Re=\emptyset e_{12}$ and $\Im / \Re \cong \emptyset e_{11} \oplus \emptyset e_{22}$ where $e_{i j}, i, j=1,2$ is the usual set of matrix units. Thus $\Im / \Re$ is associative. On the other hand, $\Im$ is not associator nilpotent since ]$\left.e_{12}, e_{11}, e_{22}\right]=\frac{1}{4} e_{12}$. This example shows also that the associator nilpotency of an ideal $\Re$ and of $\Im / \Re$ does not imply the associator nilpotency of $\Im$.

3. Analogue of Engel's theorem. If $a, b \in \mathfrak{Y}$ we write $R_{a, b}$ for $R_{a} R_{b}-R_{a . b}$ where $R_{a}$ is the linear mapping $x \rightarrow x . a$ in $\Im$. We have $x R_{a, b}=(x . a) . b-x .(a . b)$ $=[x, a, b]$. Hence if $\Im$ is associator nilpotent then there exists an integer $M$ such that $R_{a_{1}, b_{1}} R_{a_{2}, b_{2}} \cdots R_{a_{M}, b_{M}}=0$ for all $a_{i}, b_{i} \in \Im$. In particular, $R_{a, b}$ is nilpotent for all $a, b \in \mathfrak{Y}$. We shall now call $a$ an associator nilpotent element relative to $\Im$ if $a \in \Im$ and $R_{a, i, a . s}$ is nilpotent for all $i, j=0,1,2, \ldots$ If $\operatorname{dim}$

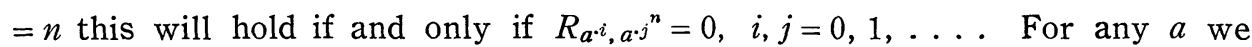
define the subspace

$$
3_{a}=\left\{z \in J \mid z\left(R_{a^{\cdot i}, a^{\cdot j}}\right)^{n}=0, i, j=0,1,2, \ldots\right\} .
$$

Then $a$ is an associator nilpotent element relative to $\Im$ if and only if $3 a=\Im$. The following result is an analogue of Engel's theorem on Lie algebras.

TheоRем 4. If $\Phi$ is infinite then $\mathfrak{\Im}$ over $\Phi$ is associator nilpotent if and only if every $a \in \mathfrak{\Im}$ is associator nilpotent relative to $\Im$.

Proof. The hypothesis amounts to assuming the identities $\left(x R_{y \cdot i,}, n_{y \cdot v}\right)^{n}=0$ in 
$\Im$ where $n$ is the dimensionality of $\Im$. We now observe that if the base field is infinite then any polynomial identity $p\left(x_{1}, x_{2}, \ldots, x_{r}\right)=0$ which is valid in a finite dimensional algebra $\mathfrak{\xi}$ is valid also for any extension algebra $\mathfrak{\Im}_{n}$. Here we let $\left(u_{1}, \ldots, u_{n}\right)$ be a basis for $\mathfrak{F}$, hence for $\Im_{n}$, and we take $x_{j}=$ $\sum \xi_{j i} u_{i}$ where $\xi_{j i}$ are indeterminates. Thus the $x_{j}$ are elements of $\mathfrak{\Im}_{\Phi\left(\xi_{j 2}\right)}$ and $p\left(x_{1}, \ldots, x_{r}\right)=\sum_{i} p_{i}\left(\xi_{j k}\right) u_{i}$. If $p\left(a_{1}, \ldots, a_{r}\right)=0$ for all $a_{j} \in \Im$ then $p_{i}\left(\alpha_{j k}\right)=0$ for all choices of the $\alpha_{j k}$ in $\emptyset$. It follows that $p_{i}\left(\xi_{j k}\right)=0$ and consequently $p\left(a_{1}, \ldots, a_{r}\right)=0$ for all $a_{j} \in \mathfrak{Y}_{\mathrm{n}}$. In particular, we see that our hypothesis on $\Im$ carries over to $\Im_{n}$ for any extension field $I / \mathscr{D}$. It therefore suffices to prove the theorem for algebraically closed $\emptyset$. Let $\Phi[a]$ be the subalgebra of $\Im$ generated by $a$ and let $\mathfrak{I}_{a}$ be the space of $\Phi$-linear combinations of the elements $R_{b, c}, b, c \in \Phi[a]$. Since every element of $\Phi[a]$ is a linear combination of the powers $a^{i}$, it is clear from the definition of $R_{b, c}$ that every $R_{b, c}$ with $b, c$ in $\Phi[a]$ is a linear combination of the operators $R_{a, i, a . j}$. We recall also the operators $R_{a, i}$ commute. Hence the operators $R_{a, i, a . j}$ commute and consequently the hypothesis implies that every $R_{b, c}$ is nilpotent if $b, c \in \Phi[a]$. Now let $e_{1}$, $e_{2}, \ldots, e_{r}$ be a set of orthogonal primitive idempotents in $\Im$ such that $e_{j} \neq 0$ and $\sum e_{i}=1$ and let $\Im=\sum_{i=j} \Im_{i j}$ be the corresponding Peirce decomposition of Э. Take $a=\sum \alpha_{i} e_{i}$ where the $\alpha_{i}$ are distinct in $\Phi$. Then the $e_{i} \in \Phi[a]$ so the foregoing result shows that $R_{e_{i}, e_{j}}$ is nilpotent. As before, let $i \neq j$ and let $a_{i j} \in$ $\Im_{i j}$. Then $a_{i j} R_{e_{i}, e_{j}}=\left[a_{i j}, e_{i}, e_{j}\right]=\frac{1}{4} a_{i j}$. Since $R_{e_{\iota}, e}$, is nilpotent this implies that $a_{i j}=0$. Hence every $\Im_{i j}=0$ for $i \neq j$ and $\Im=\sum \Im_{i i}$ is a direct sum of almost nil ideals. Hence $\Im$ is associator nilpotent by Theorem 2 .

\section{Fitting decomposition relative to an associator nilpotent subalgebra.}

We recall that if $\mathscr{L}$ is a nilpotent Lie algebra of linear transformations acting in an $n(<\infty)$ dimensional vector space $\mathfrak{M}$ then we have the Fitting decomposition $\mathfrak{M}=\mathfrak{M}_{0} \oplus \mathfrak{M}_{1}$ relative to $\mathfrak{L}$ where $\mathfrak{M}_{0}=\left\{z \mid z A^{n}=0, A \in \mathfrak{L}\right\}$ and $\mathfrak{M}_{1}=\cap \mathfrak{M}^{* i}$ where $\mathfrak{Q}^{*}$ is the ideal generated by $\mathfrak{Q}$ in the enveloping associative algebra $\mathfrak{E}(\mathfrak{L})$ of $\mathfrak{L}$. $\mathfrak{M}_{0}$ and $\mathfrak{M}_{1}$ are called respectively the Fitting null and one component of $\mathfrak{M}$ relative to $\mathfrak{L}$ (Jacobson, Lie Algebras, p. 39). These are invariant subspaces relative to $\mathfrak{L}$ (and $\mathfrak{Q}^{*}$ ) and $\mathfrak{M}_{0}\left(\mathfrak{Q}^{*}\right)^{n}=0$. We remark that $\mathfrak{M}_{1}$ can be characterized as any complementary subspace of $\mathfrak{M}$, which is invariant under $\mathfrak{2}$. For, if $\mathfrak{N}$ is such a complement then $\mathfrak{M}=\mathfrak{M}_{0} \oplus \mathfrak{N}$ and $\mathfrak{N}$ has the Fitting decomposition $\mathfrak{N}_{0} \oplus \mathfrak{N}_{1}$ where $\mathfrak{N}_{0}$ is the Fitting null component of $\mathfrak{N}$ 
relative to $\mathfrak{L}$. Since $\mathfrak{M}_{0}$ is the Fitting null component of $\mathfrak{M}$ relative to $\mathfrak{L}, \mathfrak{\Re}_{0}=0$ so $\mathfrak{R}=\mathfrak{R}_{1} \subseteq \mathfrak{M}_{1}$ by the definition of the 1-components. It follows from the direct decompositions that $\mathfrak{R}=\mathfrak{M}_{1}$. Let $\Pi$ be an extension of the base field $\Phi$ of $\mathfrak{M}$ and $\mathfrak{R}$. Then $\mathfrak{L}_{n}$ is a nilpotent Lie algebra of linear transformations in $\mathfrak{M}_{\Pi}$. Since $\mathfrak{M}_{0}\left(\mathfrak{R}^{*}\right)^{n}=0$ and every element of $\left(\mathfrak{R}_{n}\right)^{*}$ is a $P$-linear combination of the elements of $\mathfrak{L}^{*}$ it is clear that $\mathfrak{M}_{0 n}$ is the Fitting null component of $\mathfrak{M}_{n}$ relative to $\mathfrak{L}_{n}$. It is clear also that $\mathfrak{R}_{1 \mathrm{n}}$ is the Fitting one component of $\mathfrak{M}_{n}$ relative to $\mathfrak{\Omega}_{\mathrm{n}}$.

Now let $\mathscr{R}$ be a subalgebra of the Jordan algebra $\Re$. We let $\mathcal{L}_{\mathfrak{\Im}}(\mathscr{R})$ denote the Lie algebra of linear transformations in $\Im$ generated by the mappings $R_{b, c}$, $b, c \in \Re$. We have the following theorem.

THEOREM 5. Let $\Omega$ be an associator nilpotent subalgebra of a Jordan algebra $\Im$. Then the Lie algebra of linear transformations $\Omega_{\mathfrak{S}}(\Omega)$ is nilpotent and if $\mathfrak{\Im}=$ $\Im_{0} \oplus \Im_{1}$ is the Fitting decomposition of $\mathfrak{F}_{\text {relative to }} \mathfrak{L}_{\mathfrak{S}}(\mathfrak{\Re})$ then $\mathfrak{\Im}_{0}$ is a subalgebra and $\mathfrak{\Im}_{0} \cdot \mathfrak{\Im}_{1} \subseteq \Im_{1}$. Moreover, if the base field is algebraically closed then $\Im_{0}=\bigcap_{b \in \Re} 3_{\mathfrak{R}}$ where $3 a$ is defined by (11).

Proof. It is clear from our remarks that it is enough to prove the result assuming the base field $\emptyset$ is algebraically closed. Then, by Theorem $2, \Re=\sum \oplus \Omega_{i}$ where $\Re_{i}=\emptyset e_{i}+\Re_{i}$ is an ideal in $\Re_{\text {and }} e_{i}$ is the identity of $\Re_{i}$. Then $\mathfrak{R}=\sum \mathfrak{N}_{i}$ is the radical of $\Re$. Hence $R_{z}, z \in \mathfrak{N}$, is in the radical of the enveloping associative algebra of the linear transformations $R_{b}, b \in \Re$, acting in $\Im$. It follows that $R_{z, b}, z \in \Re, b \in \Re$, is in this radical. Consequently, the mappings $R_{z, b}, z \in \mathfrak{R}, b \in \Re$, are in the radical $\Subset$ of the enveloping associative algebra $\mathfrak{F}\left(\mathfrak{L}_{\mathfrak{S}}(\mathfrak{I})\right)$ of the Lie algebra $\mathfrak{L}_{\mathfrak{S}}(\Re)$. Let $\mathfrak{\Im}=\sum_{i \leq j} \Im_{i j}$ be the Peirce decomposition of $\Im$ relative to the $e_{i}\left(1=\sum e_{i}\right)$. Then $\Re_{i} \subseteq \Im_{i i}$ and every $\Im_{i j}$ is an invariant subspace of $\Im$ relative to the $R_{b}, b \in \Re=\sum \Re_{i}$. Hence to prove that $\Omega_{\mathfrak{S}}(\Re)$ is a nilpotent Lie algebra of linear transformations it suffices to show that for every $i, j$ the restrictions $R_{b, c}^{i j}$ of $R_{b, c}, b, c$ in $\Re$, generate a nilpotent Lie algebra of linear transformations acting in $\Im_{i j}$. Write $b=\sum_{k}\left(\beta_{k} e_{k}+z_{k}\right), c=\sum_{n}\left(\gamma_{k} e_{k}+w_{k}\right)$, $\beta_{k}, \gamma_{k} \in \mathscr{D}, z_{k}, w_{k} \in \mathfrak{N}_{k}$. Then $R_{b, c}=\sum \beta_{k} \gamma_{l} R_{e_{k}, e_{l}}+S$ where $S \in \cong$. We have $R_{b, c}^{i j}=\sum \beta_{k} r_{l} R_{\epsilon_{k}, e_{l}}^{i j}+S^{i j}$ where, in general, $A^{i j}$ denotes the restriction of $A$ to $\Im_{i j}$. One checks that $R_{e_{k}, e_{l}}^{i j}$ is a scalar. Hence $R_{b, c}^{i j}=\mu_{i j} 1^{i j}+S^{i j}$ where $\mu_{i j} \in \emptyset$. Since the $S_{i j}$ are contained in a nilpotent ideal of $\mathbb{E}\left(\mathscr{Q}_{\mathfrak{S}}(\Re)\right)^{i j}$ it is clear that 
the $R_{b, c}^{i j}$ generate a nilpotent Lie algebra of linear transformations acting in $\Im_{i j}$. This proves that $\mathfrak{L}_{\mathfrak{S}}(\Re)$ is nilpotent. We now observe that $R_{e_{k}, e_{l}}^{i i}=0$ so $R_{b, c}^{i i}=S^{i i} \in \mathcal{S}^{i i}$. Since $S^{i i}$ is nilpotent it follows that $\Im_{i i} \subseteq \Im_{0}$ the Fitting null component of $\Im$ relative to $\Omega_{\Im}(\Re)$. Hence $\sum \Im_{i i} \subseteq \Im_{0}$. Since $\bigcap_{b \in \Re} \Re_{b} \supseteq \Im_{0}$ we have $\bigcap_{b \in \Omega} 3 b \supseteq \Im_{0} \supseteq \sum \Im_{i i}$. Next choose $b=\sum \beta_{i} e_{i}$ so that the $\beta_{i}$ are distinct. Then $\mathfrak{\Im}=\sum_{i=j} \Im_{i j}$ is a decomposition of $\mathfrak{\Im}$ into $\mathfrak{L}_{\mathfrak{S}}(\Phi[b])$ invariant subspaces. Since $e_{i} \in \Phi[b]$ so that $R_{e_{i}, e_{j}} \in \mathfrak{L}_{\mathfrak{S}}(\Phi[b])$ and $\mathfrak{\Im}_{i j} R_{e_{i}, e_{\jmath}}=\mathfrak{\Im}_{i j}$ if $i \neq j, 3 j \subseteq \sum \mathfrak{\Im}_{i i}$. Hence $\bigcap_{b \in \mathscr{\Re}} 3_{b} \subseteq \sum \Im_{i i}$. This and the earlier inclusion imply that $\bigcap_{b \in \mathscr{R}} 3_{j}=\Im_{0}=\sum \Im_{i i}$. Since $\sum_{i<j} \mathfrak{\Im}_{i j}$ is invariant under $\mathfrak{S}_{\mathfrak{S}}(\Re)$ and $\mathfrak{F}=\mathfrak{\Im}_{0} \oplus \sum_{i<j} \mathfrak{\Im}_{i j}$, we see that $\sum_{i=j} \mathfrak{\Im}_{i j}$ is the Fitting one component of $\Im$ relative to $\mathfrak{L}_{\mathfrak{S}}(\Re)$. Thus $\mathfrak{\Im}_{0}=\sum \mathfrak{\Im}_{i i}$ is a subalgebra and $\mathfrak{\Im}_{0} \cdot \mathfrak{I}_{1} \subseteq \Im_{1}$ follows from the properties of the Peirce decomposition.

5. Cartan subalgebras of Jordan algebras. We have noted $(\S 2)$ that if $\Im$ is an associator nilpotent Jordan algebra then there exists an integer $M$ such that $R_{a_{1}, b_{1}} R_{a_{2}, b_{2}} \cdots R_{a_{M}, b_{M}}=0$ for $a_{i}, b_{i} \in \Im$. It follows that $\Omega_{\mathfrak{J}}(\Im)$ is nilpotent (cf. Theorem 5 ) and $\mathfrak{F}$ coincides with the Fitting null component of $\mathfrak{\Im}$ relative to $\mathfrak{S}(\Im)$. This implies that if $\mathscr{R}$ is an associator nilpotent subalgebra of an arbitrary Jordan algebra $\Im$ then $\mathscr{R} \subseteq \Im_{0}$ the Fitting null component of $\Im$ relative to the Lie algebra $\mathbb{S}_{\mathfrak{S}}(\Re)$. We now give the following

Definitions. A Cartan subalgebra of a Jordan algebra $\Im$ is an associator nilpotent subalgebra $\Re$ of $\Im$ such that $\Re=\Im_{0}$ the Fitting null component of $\Im$ relative to $\mathscr{R} \mathfrak{S}(\mathscr{\Omega})$. An element $a \in \mathfrak{J}$ is associator regular in $\mathfrak{I}$ if $\operatorname{dim} 3 a$ is minimal.

The proofs of Theorem 5 and Theorem 2 show that if the base field is algebraically closed then $\Re$ is a Cartan subalgebra of $\Im$ if and only if $\Re=\sum \Im_{i i}$ where $\Im=\sum_{i=j} \Im_{i j}$ is the Peirce decomposition of $\Im$ relative to a set of non-zero primitive orthogonal idempotents $e_{i}$ such that $\sum e_{i}=1$. Hence the existence of Cartan subalgebras is clear in the algebraically closed case. We shall need a stronger result on the imbedding of associator regular elements in Cartan subalgebras.

Let $\left(u_{1}, u_{2}, \ldots, u_{n}\right)$ be a basis for $\Im / \emptyset, \Xi=\emptyset\left(\xi_{1}, \xi_{2}, \ldots, \xi_{n}\right)$ the field of rational expressions in $n$ indeterminates $\xi_{i}$. The element $x=\sum \xi_{i} u_{i}$ of $\xi_{\Xi}$ is called a generic element of $\Im$. Let $M^{i j}(x)$ denote the $n \times n$ matrix of the linear transformation $R_{x . i_{. x} \cdot{ }^{n}}$ in $\Im_{\Xi}$ relative to the basis $\left(u_{1}, u_{2}, \ldots, u_{n}\right)$. The entries of $M^{i j}(x)$ are polynomials in the $\xi$ 's and the same is true of the 
matrix $M(x)$ of $n$ columns which is obtained by writing the $M^{i j}(x)$ in a single column of $n \times n$ matrices in some order. Let $r$ be the rank of $M(x)$ and set $l=n-r$. Then $l$ is the dimensionality of the subspace $3_{x}$ in $\Im_{\Xi}$ (cf. (11)) and it is clear that $l \geq 1$ since $1 \in 3 x$. Thus $r<n$. If $a \in \mathcal{G}, a=\sum \alpha_{i} u_{i}, \alpha_{i} \in \mathscr{D}$, and the rank $r^{\prime}$ of the matrix $M(a)$ obtained by the specialization $\xi_{i} \rightarrow \alpha_{i}$ in $M(x)$ does not exceed $r$. Hence $\operatorname{dim} 3 a=n-r^{\prime} \geq l$. If $\Phi$ is infinite we can choose the $\alpha$ 's so that $r^{\prime}=r, \operatorname{dim} 3 a=l$. Hence in this case $a$ is associator regular if and only if $\operatorname{dim} 3_{a}=l$. Also we see that these elements form a Zariski open subset of $\Im$. We can now prove

THEOREM 6. If $a$ is an associator regular element of a Jordan algebra $\mathfrak{Y}$ over an infinite field $\Phi$ then $B_{a}$ is a Cartan subalgebra of $\Im$ containing $a$ ).

Proof. Let $a$ be an associator regular element of $\mathfrak{Y}$ and consider the associative, hence associator nilpotent sub-algebra $\mathscr{a}[a]$ of $\Im$. It is clear that

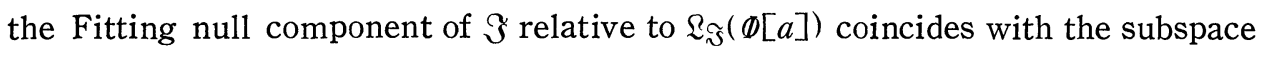
$3 a$. Hence we have a Fitting decomposition $\mathfrak{J}=3_{a} \oplus \mathfrak{Y}_{1}$ where $\mathfrak{\Im}_{1}$ is the Fitting one component of $\Im$ relative to $\mathcal{L}_{\mathfrak{J}}(\mathscr{\Phi}[a])$. Suppose the basis $\left(u_{1}, u_{2}, \ldots, u_{n}\right)$ for $\Im$ is chosen so that $\left(u_{1}, u_{2}, \ldots, u_{l}\right)$ is a basis for $3 a$ and $\left(u_{l+1}, \ldots, u_{n}\right)$ is a basis for $\Im_{1}$. Since $3_{a}$ is a subalgebra of $\Im_{\text {and }} \Im_{1} \cdot 3_{a} \subseteq \Im_{1}$ the matrices $M^{i j}(b), b \in 3 a$, have the form

$$
\left(\begin{array}{cc}
N^{i j}(b) & 0 \\
0 & P^{i j}(b)
\end{array}\right)
$$

where $N^{i j}(b)$ is the matrix of the restriction of $R_{b \cdot i, b \cdot 0^{n}}$ to $3 a$ and $P^{i j}(b)$ is the matrix of the restriction of $R_{b \cdot i, b \cdot j}{ }^{n}$ to $\Im_{1}$ relative to the indicated bases. We have $N^{i j}(a)=0$ and the rank of the matrix $P(a)$ which is a column of the matrices $P^{i j}(a)$ is $n-l$. We shall now show that every $N^{i j}(b)=0, b \in 3 a$. Suppose this is not the case and let $b$ be an element of $3 a$ such that one of the matrices $N^{i j}(b)$ has a non-zero entry $\rho$. Consider the elements $\xi a+\eta b$ in $\Im_{\Phi(\xi, \eta)}, \xi, \eta$ indeterminates. Choose a non-zero minor of order $n-l$ in $P(a)$ and consider the same minor for $\xi a+\eta b$. Its value is a polynomial $f_{1}(\xi, \eta)$ such that $f_{1}(1,0) \neq 0$. Also the entry in the column of matrices $N^{i j}(\xi a+\eta b)$ which is in the same position as $\rho$ is a polynomial $f_{2}(\xi, \eta)$ such that $f_{2}(0,1)$ $=\rho \neq 0$. Hence $f_{1}(\xi, \eta) f_{2}(\xi, \eta) \neq 0$ and we can choose $\alpha, \beta \in \Phi$ such that $f_{1}(\alpha, \beta) f_{2}(\alpha, \beta) \neq 0$. Then it is clear that if $c=\alpha a+\beta b$ then the rank of $M(c)$ 
exceeds $n-l$, contrary to the regularity of $a$. Hence we have proved that $N^{i j}(b)=0$ for every $b \in 3 a$ and every $i, j=0,1,2, \ldots$ It follows from Theorem 4 that $3 a$ is an associator nilpotent subalgebra of $\Im$. Since $a \in 3 a$ it is clear that the Fitting null component of $\Im$ relative to $\Omega_{a}(3 a)$ which is $\cap 3 b$ is contained in $b \in 3_{a}$

$3 a$. Since the reverse inequality is general we see that $3 a$ coincides with the Fitting null component relative to $\mathfrak{S}_{\mathfrak{S}}\left(B_{a}\right)$. Hence $Z_{a}$ is a Cartan subalgebra.

6. Conjugacy of Cartan subalgebras. We recall that if $a, b$ are in a Jordan algebra $\Im$ then $D_{a, b}=\left[R_{a} R_{j}\right]$ is a derivation (Jacobson [1], p. 867). Hence the linear mappings of the form $\sum D_{a_{i}, b_{i}}$ are derivations. We call such derivations inner. These constitute an ideal $\mathfrak{D}^{\prime}$ in the derivation algebra $\mathfrak{I}(\mathfrak{Z})$. We recall also that if the base field is of characteristic zero and $\mathbb{Z}$ is any Lie algebra of linear transformations in a finite dimensional vector space then the Lie algebra $\tilde{\mathfrak{l}}$ of the intersection $G$ of all the algebraic groups of linear transformations whose Lie algebras contain $\mathfrak{Q}$ satisfies $\widetilde{\mathfrak{Q}} \supseteq \mathfrak{Q}$ (Chevalley [1] II, pp. 158-169, Hochschild [1]). Evidently $G$ is an irreducible algebraic group. In particular, let $I$ be the algebraic group determined in this way by the Lie algebra $\mathscr{D}^{\prime}$ of inner derivations of $\Im$. Since the group $A$ of automorphisms of $\Im$ is an algebraic group whose Lie algebra is $\mathscr{D}(\Im)$ (Chevalley [1], II, p. 179) it is clear that $I \subseteq A$. Hence the elements of $I$ are automorphisms. We shall call these the inner automorphisms of the Jordan algebra $\Im$ (for characteristic 0 only). We shall now prove the following analogue of classical conjugacy theorem for Cartan subalgebras of Lie algebras.

TheOREM 7. Let $\Im$ be a finite dimensional Jordan algebra (with 1) over an algebraically closed field of characteristic 0 . Then if $\AA_{1}$ and $\Omega_{2}$ are Cartan subalgebras of $\mathfrak{\Im}$ there exists an inner automorphism s of $\Im$ such that $\AA_{1}^{s}=\Re_{2}$.

Proof. Our proof will be patterned after Chevalley's proof of the Lie algebra result (Chevalley [1] III, pp. 215-219). We show first that the orbit $\Omega_{1}$ of $\Omega_{1}$ under $I$ contains a Zariski open subset of $\Im$. We note first that since $\Re_{1}$ and $I$ are irreducible, $\Omega_{1}$ is épais, that is, it is irreducible and contains a non-vacuous open subset of its Zariski closure (Prop. 3, p. 193 of Chevalley [1], III). Hence our assertion will follow by showing that the dimensionality of the irreducible set $\Omega_{1}$ is $n=\operatorname{dim} \Im$. We shall do this by showing that there exists an open 
subset of $\Re_{1}$ such that the tangent space $\mathfrak{I}$ to $\Omega_{1}$ at any $a$ in this set is $\Im$. It is known that the tangent space $\mathfrak{I}$ to $\Omega_{1}$ at any $a \in \Omega_{1}$ contains $\Omega_{1}+a \mathscr{D}^{\prime}$ where $a \mathfrak{D}^{\prime}$ is the set of images of the element $a$ under the elements $D \in \mathfrak{D}^{\prime}$ (Chevalley [1], III Prop. 2, p. 192). Now, we have a set of orthogonal idempotents $e_{1}$, $e_{2}, \ldots, e_{r}$ such that if $\Im=\sum_{i \leq j} \Im_{i j}$ is the corresponding Peirce decomposition, then $\Re_{1}=\sum \Im_{i i}$. Also $\Im_{i i}=\emptyset e_{i}+\Re_{i}$ where $\Re_{i}$ is a nil ideal in $\Im_{i i}$. Let $0_{1}$ be the open subset of $\Re_{1}$ of elements $a=\sum_{1}^{r} \alpha_{i} e_{i}+z_{i}, \alpha_{i} \in \emptyset, z_{i} \in \Re_{i}$, such that $\prod_{i \neq j}\left(\alpha_{i}-\alpha_{j}\right) \neq 0$. Let $a \in 0_{1}$ and consider $a\left[R_{e_{k}} R_{a_{k l}}\right]$ where $k \neq l$ and $a_{k l} \in \mathfrak{Y}_{k l}$. We have

$$
\begin{aligned}
a[ & \left.R_{e_{k}} R_{a_{k l}}\right]=\left(\sum \alpha_{i} e_{i}+z_{i}\right)\left[R_{e_{k}} R_{a_{k l}}\right] \\
& =\left(\alpha_{k} e_{k}+z_{k}\right)\left[R_{e_{k}} R_{a_{k l}}\right]+\left(\alpha_{l} e_{l}+z_{l}\right)\left[R_{e_{k}} R_{a_{k l}}\right] \\
& =\frac{1}{4}\left(\alpha_{k}-\alpha_{l}\right) a_{k l}+\frac{1}{2}\left(z_{k}-z_{l}\right) . a_{k l} .
\end{aligned}
$$

Hence the tangent space $\mathfrak{I}$ to $\Omega_{1}$ at a contains $\left(\alpha_{k}-\alpha_{l}\right) a_{k l}+2\left(z_{k}-z_{l}\right) . a_{k l}=a_{k l} S_{k l}$ where $S_{k l}$ is the linear operator $\left(\alpha_{k}-\alpha_{l}\right) 1+R_{2\left(z_{k}-z_{l}\right)}$. It is clear from the multiplication table (4) for Peirce spaces that $S_{k l}$ maps $\Im_{k l}$ into itself. Also $z_{k}-z_{l}$ is nilpotent, hence $R_{2\left(z_{k}-z_{l}\right)}$ is nilpotent (Albert [2], p. 550). Since $\alpha_{k}$ $-\alpha_{l} \neq 0$ it is clear that $S_{k l}$ has an inverse. Hence $S_{k l}$ maps $\Im_{k l}$ onto itself and consequently $\mathfrak{I}$ contains $\mathfrak{J}_{k l}$. It now follows that $\mathfrak{I}=\mathfrak{F}$. Since $\Omega_{1}$ is the orbit of $\Omega_{1}$ it is clear that $\Omega_{1}$ contains a simple point of $\Omega_{1}$. Since $\Omega_{1}$ is irreducible the open subset $0_{1}$ contains a simple point. Hence $\operatorname{dim} \Omega_{1}=n$ and $\Omega_{1}$ contains a non-vacuous open subset of $\Im$. In the same manner we have that the orbit $\Omega_{2}$ of the Cartan subalgebra $\Omega_{2}$ contains a non-vacuous open set. Since the set of associator regular elements is Zariski dense we see that $\Omega_{1} \cap \Omega_{2}$ contains an associator regular element $b$. Hence there exist inner automorphisms $s_{1}, s_{2}$ such that $b \in \Re_{i}^{s_{i}}$. Then $3 b \supseteq \Re_{i}^{s_{i}}$ and since $3 b$ and $\Re_{i}^{s_{i}}$ are Cartan subalgebras, $乃_{b}=\Re_{1}^{s_{1}}=\Re_{2}^{s_{2}}$. Hence $\Re_{2}=\Re_{1}^{s}$ where $s=s_{1} s_{2}^{-1} \in I$.

7. Applications to generic traces. Separability criterion. As in $\S 5$, let $x=\sum_{1}^{n} \xi_{i} u_{i}$ be a generic element of $\Im$ over $\Phi$ where $\left(u_{1}, u_{2}, \ldots, u_{n}\right)$ is a basis and the $\xi$ 's are indeterminates. Then one knows that the minimum polynomial $m_{x}(\lambda)$ of $x$ in $\Im_{\Xi}, \Xi=\emptyset\left(\xi_{1}, \ldots, \xi_{n}\right)$, has the form $\lambda^{m}-\sigma_{1}(\xi) \lambda^{m-1}+\cdots+$ $(-1)^{m} \sigma_{m}(\xi)$ where $\sigma_{j}(\xi) \equiv \sigma_{j}\left(\xi_{1}, \ldots, \xi_{n}\right)$ is a homogeneous polynomial of degree $j$ in the $\xi$ 's. If $a=\sum \alpha_{i} u_{i} \in \mathfrak{F}$ where the $\alpha_{i} \in \emptyset$ then the specialization $\xi_{i} \rightarrow \alpha_{i}$ gives a polynomial $m_{a}(\lambda)=\lambda^{m}-\sigma_{1}(\alpha) \lambda^{m-1}+\cdots$ such that $m_{a}(a)=0$. The 
polynomial $m_{a}(\lambda)$ is called the generic minimum polynomial of $a, T(a) \equiv \sigma_{1}(\alpha)$ and $N(a) \equiv \sigma_{m}(\alpha)$ are the generic trace and norm respectively of $a$. The degree $m$ of $m_{x}(\lambda)$ is called the degree of the algebra. The mapping $a \rightarrow T(a)$ is linear and the bilinear form $T(a, b)=T(a . b)$, which is called the generic trace form, is symmetric and associative in the sense that $T(a . b, c)=T(a, b . c$ ) (Tits $[1]$, p. 35). The generic minimum polynomial (hence the trace and norm) are unchanged under extension of the base field. If $\Phi$ is infinite then the set of elements $a$ such that the generic minimum polynomial $m_{a}(\lambda)$ coincides with the minimum polynomial $\mu_{a}(\lambda)$ of $a$ is a non-vacuous Zariski open subset of $\Im$. The same is true of the subset of associator regular elements. Hence Theorem 6 implies that for infinite base fields there exist Cartan sub-algebras satisfying the hypotheses of the following

Theorem 8. Let $\Im$ be a Jordan algebra over an algebraically closed field $\Phi$ and let $\Re$ be a Cartan subalgebra of $\Im$ which contains an element which is associator regular and has generic minimum polynomial equal to its minimum polynomial. Suppose $\mathfrak{R}=\sum \oplus \mathfrak{R}_{i}$ where $\Re_{i}$ is an ideal in $\mathfrak{R}$ of the form $\square e_{i}+\Re_{i}$ where $e_{i}$ is the identity element of $\Re_{i}$ and $\Re_{i}$ is a nil ideal in $\Re_{i}$. Let $\Im=\Re_{1} \oplus \Im_{1}$ be the Fitting decomposition of $\mathfrak{\Im}$ relative to $\mathfrak{S}_{\mathfrak{F}}(\mathfrak{R})$. If $a \in \mathfrak{\Im}$ we write $a=\sum_{1}^{r}\left(\alpha_{i} e_{i}+z_{i}\right)+a_{1}$ where $\alpha_{i} \in \Phi, z_{i} \in \mathfrak{N}_{i}$ and $a_{1} \in \mathfrak{\Im}_{1}$. Then the generic trace

$$
T(a)=\sum n_{i} \alpha_{i}
$$

where $n_{i}$ is the maximum index of nilpotency of the elements of $\dot{\mathfrak{A}}_{i}$. Also the degree of $\Im$ is $\sum n_{i}$.

Proof. If $b \in \Re$ let $m_{b, \Re}(\lambda)$ be the generic minimum polynomial of $b$ as element of $\Re$. Then $m_{b, \Re}(\lambda)$ is a factor of $m_{b}(\lambda)$, and since $\Re_{\text {contains elements }}$ $c$ such that $m_{c}(\lambda)=\mu_{c}(\lambda)$, it follows that $m_{b, \Omega}(\lambda)=m_{b}(\lambda)$. If $b=\sum\left(\alpha_{i} e_{i}+z_{i}\right)$ and the minimum polynomial of $z_{i}$ is $\lambda^{m_{i}}$ then it is clear that $\mu_{\dot{b}}(\lambda)=$ L.C.M. $\left(\lambda-\alpha_{i}\right)^{m_{i}}$. If we introduce a generic element for $\Omega$ relative to an appropriate basis it follows easily that $m_{b, \Omega}(\lambda)=\Pi\left(\lambda-\alpha_{i}\right)^{n_{\imath}}$ where $n_{i}$ is the maximum index of nilpotency of the elements of $\mathfrak{\Re}_{i}$. Hence the generic trace of $b$ relative to $\Re, T_{\Re^{\prime}}(b)=\sum n_{i} \alpha_{i} . \quad$ Since $m_{b, \Re}(\lambda)=m_{b}(\lambda)$ we have $T(b)=\sum n_{i} \alpha_{i} . \quad$ Next we recall that $\Im_{1}=\sum_{i<j} \Im_{i j}$ where $\Im=\sum_{i \leq j} \Im_{i j}$ is the Peirce decomposition of $\Im$ relative to the $e_{i}$. Also we have seen that if $a_{i j} \in \Im_{i j}, i \neq j$, then $a_{i j}$ is an associator. Hence $T\left(a_{t j}\right)=0$ since $T(a, b)=T(a . b)$ is an associative form. It follows that 
if $a=\sum_{1}^{r}\left(\alpha_{i} e_{i}+z_{i}\right)+a_{1}, a_{1}$ in $\Im_{1}$, then $T\left(a_{1}\right)=0$. Then $T(a)=T(b), b=\sum\left(\alpha_{i} e_{i}\right.$ $\left.+z_{i}\right)$ and so $T(a)=\sum n_{i} \alpha_{i}$.

If the base field is of characteristic 0 then the conjugacy theorem implies that every Cartan subalgebra $\Re$ of a Jordan over a field of characteristic 0 satisfies the hypothesis of Theorem 8 . We conjecture that this holds also for Jordan algebras over infinite fields of characteristic $p \neq 0$.

We recall that a Jordan algebra $\Im$ is called separable if $\Im_{u}$ is semi-simple for every extension field $\Pi$ of the base field $\Phi$ of $\Im$. It is known that $\Im$ is separable if and only if the generic trace bilinear form $T(a, b)$ is non-degenerate (Jacobson [6], p. 41). The proof of the sufficiency of this condition is a general one. On the other hand, the known proof of the necessity is based on the classification of simple Jordan algebras over an algebraically closed field and consists of a case by case verification for these. We shall now give a general proof of this half of the theorem based on the formula of Theorem 8 and on a notion of a reduced Jordan algebra and reduced trace which we now define.

We shall call a Jordan algebra $\mathfrak{Y}$ reduced if $\mathfrak{G}$ contains a Cartan subalgebra $\Re$ which is a direct sum of almost nil ideals. Then $\Re=\sum_{1}^{r} \oplus \mathscr{R}_{i}$ where $\Omega_{i}$ is an ideal of the form $\Phi e_{i}+\mathfrak{N}_{i}, e_{i}^{\cdot 2}=e_{i}, \mathfrak{N}_{i}$ is a nil ideal in $\Re_{i}$. If $\mathfrak{\Im}=\mathfrak{\Re} \oplus \mathfrak{Y}_{1}$ is the Fitting decomposition of $\mathfrak{\Im}$ relative to $\mathbb{Q}_{\mathfrak{F}}(\mathfrak{R})$ and $a=\sum_{i}^{r}\left(\alpha_{i} e_{i}+z_{i}\right)+a_{1}, z_{i} \in$ $\mathfrak{N}_{i}, a_{1} \in \mathfrak{Y}_{1}$, then we define the reduced trace $t(a)=\sum_{1}^{r} \alpha_{i}$. Clearly, $a \rightarrow t(a)$ is a linear function on $\Im$. We wish to show that $t$ vanishes on all associators, or, equivalently, $t(a, b)=t(a . b)$ is an associative bilinear form on $\Im$. As in the algebraically closed case (proof of Theorem 5 ), $\mathfrak{I}_{1}=\sum_{i<j} \mathfrak{\Im}_{i j}$ where $\mathfrak{\Im}=\sum_{i=j} \Im_{i j}$ is the Peirce decomposition of $\Im$ relative to the $e_{i}$.

LemmA. If $a_{i j} \in \Im_{i j}, i \neq j$, then $a_{i j}^{2}=\alpha\left(e_{i}+e_{j}\right)+z_{i}+z_{j}$ where $\alpha \in \Phi, z_{k} \in \mathfrak{N}_{k}$.

Proof. We have $a_{i j}^{2} \in \Im_{i i}+\Im_{j j}$ so $a_{i j}^{2}=\alpha e_{i}+\beta e_{j}+z_{i}+z_{j}, \alpha, \beta \in \emptyset, z_{k} \in \mathfrak{N}_{k}$. Then $\left(a_{i j}^{2} \cdot e_{i}\right) \cdot a_{i j}=a_{i j}^{2} \cdot\left(e_{i} \cdot a_{i j}\right)$ gives

$$
\left(\alpha e_{i}+z_{\imath}\right) \cdot a_{i j}=\frac{1}{2}\left(\alpha e_{i}+\beta e_{j}+z_{i}+z_{j}\right) \cdot a_{i j}
$$

Hence $\left(\alpha e_{i}-\beta e_{j}\right) \cdot a_{i j}=\left(z_{j}-z_{i}\right) . a_{i j}$ so $2\left(z_{j}-z_{i}\right) . a_{i j}=(\alpha-\beta) a_{i j}$. Since $z_{j}-z_{i}$ is nilpotent, $R_{z_{j}-z_{i}}$ is nilpotent. Hence the last equation gives $(\alpha-\beta)^{n} a_{i j}=0$ for 
some positive integer $n$. Then $\alpha=\beta$ since this holds also if $a_{i j}=0$. Thus $a_{i j}^{2}=\alpha\left(e_{i}+e_{j}\right)+z_{i}+z_{j}$.

We can now prove

TheOREM 9. The reduced trace from $t(a, b)=t(a . b)$ is associative. ${ }^{5)}$

Proof. We have to show that $t([a, b, c])=0$ for all $a, b, c \in \Im$. It is enough to prove this for $a, b, c$ in the Peirce spaces $\Im_{i j}$ relative to the $e_{i}$. Now $t\left(a_{i j}\right)$ $=0$ if $a_{i j} \in \Im_{i j}, i \neq j$, and the product of three elements of Peirce spaces is 0 or is in a $\Im_{i j}, i \neq j$, except in the following cases: $\mathrm{I}$. the three elements are in an $a_{i i}$, II. two of the elements are in a $\Im_{i j}, i \neq j$, and the third is in $\Im_{i i}$, III. one element is in $\Im_{i j}$, the second in $\Im_{j k}$ and the third in $\Im_{i k}$ where $i, j, k$ are unequal. We note also that in any commutative algebra one has the "Jacobi identity": $[a, b, c]+[b, c, a]+[c, a, b]=0$ for associators. Hence it suffices to verify that $t([a, b, c)]-0$ in the following cases:

$$
\begin{array}{cl}
\text { I. } & t\left(\left[a_{i i}, b_{i i}, c_{i i}\right]\right)=0, a_{i i}, b_{i i}, c_{i i} \in \Im_{i i} \\
\text { II'. }^{\prime} & t\left(\left[a_{i j}, b_{i j}, c_{j j}\right]\right)=0 \quad a_{i j}, b_{i j} \in \Im_{i j}, c_{j j} \in \Im_{j j} \\
\text { II'. }^{\prime \prime} & t\left(\left[a_{i j}, c_{j j}, b_{i j}\right]\right)=0, a_{i j}, b_{i j} \in \Im_{i j}, c_{j j} \in \Im_{j j} \\
\text { III. } & t\left(\left[a_{i j}, b_{j k}, c_{k i}\right]\right)=0, a_{i j} \in \Im_{i j}, b_{j k} \in \Im_{j k}, c_{i k} \in \Im_{i k}, i, j, k \neq .
\end{array}
$$

The first of these is clear since $\left[a_{i i}, b_{i i}, c_{i i}\right] \in \mathfrak{A}_{i}$ for $a_{i i}=\alpha e_{i}+z_{i}, b_{i i}=\beta e_{i}+z_{i}^{\prime}$, $c_{i i}=\gamma e_{i}+z_{i}^{\prime \prime}, z_{i}, z_{i}^{\prime}, z_{i}^{\prime \prime} \in \mathfrak{N}_{i}$. For $\mathrm{II}^{\prime}$ we note that we have the relation

$$
\left[a_{i j}, b_{i j}, c_{j j}\right]=\left(e_{j}-e_{i}\right) .\left(\left(a_{i j} \cdot c_{j j}\right) \cdot b_{i j}\right)
$$

which is obtained from (2) by taking $x=c_{j j}, a=a_{i j}, b=b_{i j}, c=e_{j}$. By Lemma $1, \quad\left(a_{i j} . c_{j j}\right) . b_{i j}=\alpha\left(e_{i}+e_{j}\right)+z_{i}+z_{j}, \quad z_{k} \in \Re_{\Re} . \quad$ Multiplication by $e_{j}-e_{i}$ gives $\alpha\left(e_{j}-e_{i}\right)+z_{j}-z_{i}$. The reduced trace of this element is 0 . Hence $t\left(\left[a_{i j}, b_{i j}, c_{j j}\right]\right)$ $=0$. For $\mathrm{II}^{\prime \prime}$ we use the relation

$$
\left[a_{i j}, c_{j j}, b_{i j}\right] . e_{i}=0
$$

which is obtained from (15) by multiplying by $e_{i}$. By Lemma 1, $\left[a_{i j}, c_{j j}, b_{i j}\right]$ $=\alpha\left(e_{i}+e_{j}\right)+z_{i}+z_{j}, z_{k} \in \mathfrak{N}_{k} . \quad$ This and the last equation give $\alpha=0$. Hence $t\left(\left[a_{i j}, c_{j j}, b_{i j}\right]\right)=0$. For III we note that we have

$$
\left[a_{i j}, b_{j k}, c_{k i}\right]=\left(e_{k}-e_{j}\right) .\left(\left(a_{i j}, c_{k i}\right) \cdot b_{j k}\right)
$$

5) Cf. Albert [3], p. 522 . 
which is obtained by setting $x=a_{i j}, a=c_{k i}, b=b_{j k}, d=e_{k}$ in (2). By the lemma, $\left(a_{i j} . c_{k i}\right) . b_{j k}=\alpha\left(e_{j}+e_{k}\right)+z_{j}+z_{k}, z_{l} \in \mathfrak{N}_{l}$. Then the result follows as for II'.

If $f(a, b)$ is any symmetric associative bilinear form on a Jordan algebra $\Im$ then it is clear that the radical $\mathfrak{F}^{\perp}$ of $f$ is an ideal. In particular, this holds if $\Im$ is reduced and $f=t$ the reduced trace form. It is clear from the definition of $t$ that $\sum \Re_{i} \subseteq \mathfrak{J}^{\perp}$ and that $t\left(e_{i}, e_{i}\right)=t\left(e_{i}\right)=1$ which shows that $t \neq 0$ and $\mathfrak{J}^{\perp}$ $\neq \Im$. Now suppose $\Im$ is simple over an algebraically closed field. Let $\Re$ be a Cartan subalgebra of $\mathfrak{Z}$ satisfying the hypotheses of Theorem 8 . Then the formula (14) for the generic trace $T(a)$ is valid. Also $\Re$ is a direct sum of almost nil ideals so we have the reduced trace $t(a)$ defined by the Fitting decomposition relative to $\Omega_{\mathfrak{S}}(\mathscr{\Omega})$. The relation $\Im^{\perp} \neq \Im$ for the radical $\Im^{\perp}$ relative to $t$ and the simplicity of $\Im$ imply that $\Im^{\perp}=0$. Hence $t$ is non-degenerate. Also $\sum \Re_{i}=0$ since $\sum \Re_{i} \subseteq \mathfrak{F}^{\perp}$. Hence (14) becomes $T(a)=\sum \alpha_{i}$ which coincides with the definition of $t(a)$. Hence we see that the generic trace form $T$ of a simple Jordan algebra over an algebraically closed field is non-degenerate. We can now prove

TheOREM 10. A Jordan algebra is separable if and only if the generic trace bilinear form $T$ where $T(a, b)=T(a . b)$ is non-degenerate.

Proof. We omit the proof of sufficiency since a general proof of this is given in Jacobson [6], p. 41. Also, the argument given in this reference shows that to prove the necessity it is enough to suppose $\mathfrak{Y}$ is simple over an algebraically closed field. Hence the proof is complete by what we have just shown.

\section{BibliogRAPHY}

A. A. Albert

[1] On Jordan algebras of linear transformations, Trans. Amer. Math. Soc., 59 (1946), 524-555.

[2] A structure theory for Jordan algebras, Ann. Math., 48 (1947), 546-567.

[3] A theory of power associative commutative algebras, Trans. Amer. Math. Soc., $69(1950), 503-527$.

C. Chevalley

[1] Théorie des groupes de Lie II (1951), III (1955), Act. Sci., Paris.

G. P. Hochschild

[1] On the algebraic hull of a Lie algebra, Proc. Amer. Math. Soc., 19 (1960), 195-200. N. Jacobson

[1] Derivation algebras and multiplication algebras of semi-simple Jordan algebras, Annals of Math., 50 (1949), 866-874. 
[2] General representation theory of Jordan algebras, Trans. Amer. Math. Soc., 70 (1951), 509-530.

[3] Structure of alternative and Jordan bimodules, Osaka Math. Jour., 6 (1954), 1-71.

[4] A theorem on the structure of Jordan algebras, Proc. Nat. Acad. Sci., 42 (1956), 140-147.

[5] Some groups of transformations defined by Jordan algebras I, Jour. für reine und angew. Math., 201 (1959), 178-195.

[6] Generic norm of an algebra, Osaka Math. Jour., 15 (1963), 25-50.

K. McCrimmon

[1] Jordan algebras of degree 1, Bull. Amer. Math. Soc., 70 (1964), p. 702.

A. J. Penico

[1] The Wedderburn principal theorem for Jordan algebras, Trans. Amer. Math., Soc., 70 (1951), 404-420.

j. Tits

[1] A theorem on generic norms of strictly power associative algebras, Proc. Amer. Math. Soc., 15 (1964), 35-36.

Yale University 